\title{
Commented review on refractive microlenses and microlens arrays metrology
}

\author{
Jeremy Béguelin $\odot$, Reinhard Voelkel, and Toralf Scharf* \\ SUSS MicroOptics SA, Hauterive, Switzerland
}

\begin{abstract}
The fabrication of high-quality microlenses is possible only when it is assisted by efficient and accurate metrology. For this reason, developing expertise in measurement procedures is crucial for the micro-optics manufacturer. We review and comment on this topic by first discussing what features of microlenses must be characterized and controlled. We then review the existing techniques and instruments that can be employed for this task. Finally, we detail the limitations of these different methods, we compare them, and we propose practical guidelines to setup effective metrology methodology. We believe that this paper can be an introduction to the topic but also serves as a reference document for the more experienced reader. (c) The Authors. Published by SPIE under a Creative Commons Attribution 4.0 Unported License. Distribution or reproduction of this work in whole or in part requires full attribution of the original publication, including its DOI. [DOI: 10.1117/1.JOM.1.3.030901]
\end{abstract}

Keywords: refractive micro-optics; microlens; microlens arrays; metrology; surface metrology; optical testing.

Paper 21006V received Apr. 15, 2021; accepted for publication Aug. 12, 2021; published online Aug. 31, 2021.

\section{Introduction}

Over the years, refractive micro-optics has been demonstrated to be a key component of many high-tech systems proposing various applications. Among the most significant, we can mention fiber coupling for data communication, ${ }^{1}$ laser beam shaping, ${ }^{2}$ or illumination systems. ${ }^{3}$ A common point of all these applications is the requirement of high-quality micro-optics. The achievable performance of a microlens, or a microlens array (MLA), is related to the capability of the manufacturing process, but one cannot fabricate what one cannot measure. Therefore, measurement capability represents a fundamental limit in this context. This is especially true for refractive microlenses, whose metrology is nothing like an easy and standard process.

At this point, we should define more clearly what we mean by microlens metrology. By definition, metrology is related to measurement processes; so which features of a microlens should we measure? To answer this, we first need to address the following interrogations: what pieces of information do we want to obtain, in other words, what characterization do we want to perform? Generally, the characterization has two purposes: first, the assessment of the microlens performance. One possibility is to make a functional test, i.e., measuring directly an optical merit function. The alternative is to derive the performance from the geometrical and physical parameters of the microlens. Second, the characterization is intended to provide feedback to optimize the fabrication process. In this case, one wants to obtain the geometrical features of the microlens, principally the form of its surface. In conclusion, microlens metrology is related to optical testing on the one hand and to surface metrology on the other hand.

Like manufacturing microlenses is different than manufacturing standard macroscopic lenses, microlenses metrology has also differences from its classical counterpart, the main reason being the scale difference. Two approaches can be distinguished: first, the adaption of the techniques used for regular macro-optics, usually based on optical testing such as interferometry, to micro-optics. The challenge is the manipulation of the probing beam as such a scale. The other approach is to use the surface metrology instruments that were developed for the semiconductor industry, such as wight-light interferometers or stylus profilometers. In this case, the challenge is that a microlens surface is not flat.

*Address all correspondence to Toralf Scharf, toralf.scharf@ suss.com 
Refractive microlenses metrology has been a topic of research and development as it enables the optimization of novel fabrication techniques. A series of standards from the International Organization for Standardization (ISO) ${ }^{4-8}$ was created to standardize microlenses metrology. However, it dates back to 2001, and even though it was revised, it does not take into account the most recent developments. For instance, it does not consider anything other than spherical surfaces even though microlenses are no more limited to this basic geometry. ${ }^{9}$ Freeform microlenses are slowly but certainly emerging. ${ }^{10}$ This is the consequence of novel fabrication techniques such as direct writing ${ }^{11,12}$ combined with replication ${ }^{13}$ (The 35 th International Conference on Micro- and Nano-Engineering) that allow for more flexibility in terms of surface form than the standard process using photoresist reflow and reactive ion etching (RIE). These advances represent a real challenge for metrology as it is clear that micro-optics is no longer limited to low numerical aperture (NA) spherical microlenses. Other review papers have been published ${ }^{14-16}$ but are either very general, outdated, or not focused on microlens metrology. Within this context, this paper aims to review the current challenges posed by microlenses metrology as well as the newest developments.

More specifically, we review and compare different types of measurements and discuss their limitations. For each measurement principle, we recall what piece of information is obtained and why it is useful to have it. Naturally, two measurement features are of great interest and also thoroughly discussed: the uncertainty and the range of measurable microlens geometries. A key element that made the success of refractive micro-optics a widespread technology is the waferlevel fabrication process. ${ }^{17}$ Indeed, there might be dozens of thousands of microlenses on a single wafer, thus allowing high volume production. For this reason, one also has to take into account more practical but nevertheless crucial considerations: the measurement time, automation capability, and commercial aspects. These are also fundamentally important for integration in industry and use in production. Due to our background as industrial manufacturers, this paper is influenced by our practical experience.

This paper is organized as follows: in Sec. 2, we focus on the measurement of the geometrical and physical parameters of a microlens. In Sec. 3, we cover the measurement of the optical function of a microlens. In Sec. 4, we discuss what supplementary considerations should be taken into account for arrays of microlenses. In Sec. 5, we provide a general discussion and comparison of the different approaches as well as practical guidelines. Finally, Sec. 6 provides a brief conclusion and outlook.

\section{Microlens Geometrical and Physical Parameters}

Typically, a microlens consists of a single curved surface manufactured on a substrate, usually a wafer. This can be achieved by material subtraction or additive manufacturing, in which case, two different materials must generally be considered. Figure 1 shows a schematic of a typical

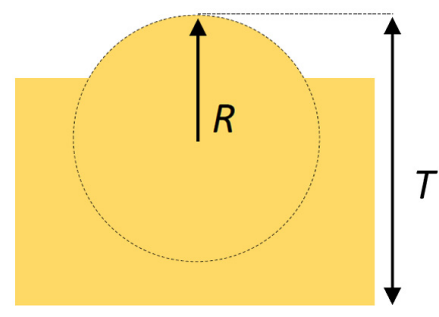

(a)

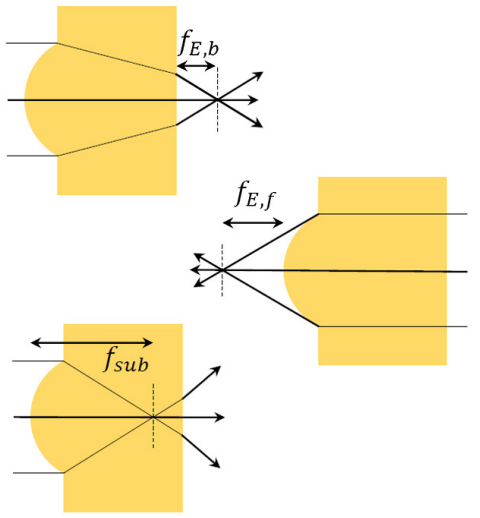

(b)

Fig. 1 Schematics of a spherical plano-convex microlens fabricated in a single substrate of thickness $T$ and with a ROC $R$ (a). Definition of the focal lengths in different configurations (b). 
plano-convex microlens made in a single substrate. Most of the time, the microlens surface presents a rotational symmetry, is elliptical or cylindrical. The optical function of a singlematerial microlens depends on three quantities: the form of its surface, the substrate thickness $T$, and the refractive index $n$. This is explicitly shown, for instance, in the expression of the effective back focal length in which $T$ appears:

$$
f_{E, b}=\frac{R}{n-1}-\frac{T}{n},
$$

with $R$ the surface radius of curvature (ROC), $T$ is the substrate thickness, and $n$ is the refractive index. Consequently, controlling all these parameters is necessary to ensure a good performance of the microlens. Thus, we discuss the impact of these three quantities in the following sections.

\subsection{Substrate Refractive Index}

The refractive index can span a large range, from about 1.44 for glass to 3.5 for silicon, and its variation is usually measured by interferometry. ${ }^{18,19}$ The quantitative evaluation of this variation is defined by standards, ${ }^{20,21}$ and materials are sorted into different classes. The three best classes available from wafer manufacturers have a refractive index variation $\Delta n<5 \cdot 10^{-6}$, giving an optical path difference peak-to-valley (PV) smaller than $5 \mathrm{~nm}$ for wafers with a thickness of $1 \mathrm{~mm}$. Such variation can thus reasonably be neglected for micro-optics with substrate thicknesses in the range of 0.5 to $5 \mathrm{~mm}$. For this reason, one can occult this control step in the microlens metrology process when high-quality substrates are used.

\subsection{Substrate Thickness}

A variation in the substrate thickness mainly changes the effective back focal length, as can be seen in Eq. (1). Controlling the substrate thickness is thus particularly important in systems where the microlens position cannot be adjusted to compensate for defocusing. For example, a tilt between the front and back surfaces of the substrate is problematic for an MLA as it creates a linear variation of the microlenses focal length.

To do so, thanks to the long history of the semiconductor industry, many different tools are commercially available to measure the substrate thickness and ensure its uniformity. Likewise, there exist many wafer suppliers. Usually, the thickness wafer tolerance is typically in the range \pm 10 to $25 \mu \mathrm{m}$ and the total thickness variation in the range 1 to $10 \mu \mathrm{m}$ depending on the material and the wafer size. Measuring and monitoring these parameters does not present any particular difficulty and is thus not the heart of the microlens metrology although it has to be done.

\subsection{Microlens Surface}

The surface of a plano-convex microlens is the functional component of a microlens and its fabrication is the main know-how of manufacturers. As the refractive index variation is negligible and the thickness variation produces only a focal shift, the performance of a microlens is mostly dictated by its surface. For this reason, the characterization of microlenses is very closely linked to surface metrology and the assessment of their performance by surface measurement is possible. More specifically, micro-optics deals with (a) spherical micro-surfaces that are very smooth.

The surface of microlenses can be divided into two qualitatively different components: ${ }^{22}$ first, the surface form or figure, ${ }^{23}$ which contains the low spatial frequencies of the surface and shapes the wavefront; and second, the roughness, which contains the high spatial frequencies and is mainly responsible for light scattering. ${ }^{24-26}$ This specific division is not absolute and there is no general admitted rule to determine the cutoff frequency between these two regimes. One approach is to use the Fresnel number to estimate the limit. ${ }^{27}$

As the measurement of high frequencies usually requires a different measuring system than measuring low frequencies, we discuss the measurement of the two components separately in the following sections. 


\subsubsection{Surface form}

The surface form deviation with respect to the nominal surface is the piece of information used to assess the optical performance as well as to optimize the fabrication process. To place the measurement of the surface form deviation in a dimensional context, and thus to estimate the required measurement accuracy, one can calculate the surface deviation value for high-quality microlenses. To do so, one can use the thin element approximation ${ }^{28}$ to estimate the wavefront aberration induced by the surface form deviation or the irregularity. ${ }^{29}$

For a microlens to be said diffraction-limited according to the Maréchal's criterion, the rootmean-square (RMS) value of the surface form deviation $\Delta Z_{r m s}$ must satisfy the following condition:

$$
\Delta Z_{r m s}<\frac{\lambda}{14(n-1)},
$$

with $\lambda$ the operating wavelength and $n$ the refractive index of the microlens. This gives an RMS deviation smaller than $71 \mathrm{~nm}$ for a glass microlens $(n \sim 1.5)$ used in the visible $(\lambda=500 \mathrm{~nm})$. This value drops to $38 \mathrm{~nm}$ for silicon microlenses $(n \sim 3.5)$ used in the near-infrared (IR) $(\lambda=1330 \mathrm{~nm})$. These numbers can be used to get an estimate of the uncertainty value of the measuring tool, as one usually requires this uncertainty to be about ten times smaller than the actual surface form deviation value.

There exist different techniques that can be used to measure the form of a refractive microoptical surface. They can be sorted into two categories: non-contact optically based instruments and mechanical contact probes or styluses.

The instruments of the first category, generally called optical surface profilers, probe the surface by optical means. Different techniques are commonly used for surface measurements: confocal microscopy, ${ }^{30}$ coherence scanning interferometry (CSI), ${ }^{31}$ or digital holography. ${ }^{32}$ These instruments have crucial assets: they possess a sufficient nanometric vertical resolution, they do no enter in contact with the surface, thus allowing them to measure deformable surfaces, they are pretty fast, typical measurement time of a microlens is in the order of $10 \mathrm{~s}$, and they can be automated for measurements at wafer level. These assets explain why these instruments are very popular among the micro-optics manufacturers.

However, these instruments also present drawbacks. Because they work in reflection and require a microscope objective, they cannot measure steep and large microlenses. Another drawback of optical surface profilers is the error arising in non-flat surface measurements. ${ }^{33,34}$ The amplitude of such error can be larger than the surface deviation that has to be measured. However, this error can be corrected at a satisfactory level. ${ }^{35,36}$ Consequently, the main drawback of these techniques is the limited capability to measure large and steep surfaces. This aspect is discussed in more detail in Sec. 5.1.

Historically, the first surface form measurements by optical means were performed by interferometry in reflection ${ }^{37}$ in the null configuration. The reflected wavefront aberration is converted into a surface form deviation. Even though this technique has excellent accuracy, it has the drawback that the measurement configuration depends strongly on the microlens geometry. Indeed, a "perfect" nominal surface must be created to serve as a reference. If this is possible for spherical surfaces as high-quality reference balls are commercially available, it is not the case for aspheric surfaces. This reason explains why the optical surface profilers mentioned previously are more popular.

On the other hand, there are mechanical styluses. ${ }^{38}$ This type of instrument has certain drawbacks that make them less popular than their optical counterparts. First, they are rather slow as they measure profiles. For rotationally symmetric microlenses, at least three scans are needed to obtain a single profile going through the vertex of the microlens. For the full surface, even more scans are needed, meaning a long measurement time. Second, the contact mode has some disadvantages. Indeed, the tip of the instrument might damage the surface. Also, certain surfaces (typically polymers) are sticky and this causes measurement artifacts, thus rendering the measurement results untrustworthy.

These drawbacks are counterbalanced by the main asset of this technique, which is the capability to measure the steep slopes of large surfaces that cannot be measured by optical means in reflection. 
For completeness, we can also mention that some instruments allow one to directly measure parameters of the surface form such as the ROC. ${ }^{37,39}$ One interesting question that has no easy answer is how this value of ROC compares with the value derived from a surface measurement through a fit process? This comparison is even more complicated if the surface is aspheric. In summary, these types of measurements give only partial information about the surface form, and results should be manipulated with care.

\subsubsection{Surface roughness}

Typical values for microlens roughness are in the range $S_{q}=1$ to $10 \mathrm{~nm}$. The vertical resolution of the measuring tool must thus be sufficient to measure this. Also, the highest spatial frequency that the instrument should be able to measure to cover all the roughness range ${ }^{26}$ must be in the order of the light working wavelength, thus about $0.5 \mu \mathrm{m}$.

For optical surface profilers, measuring the roughness is possible when they are equipped with high magnification microscope objectives $(100 \times$ or higher). Indeed, this allows accessing high frequencies components. Moreover, it is only with these magnifications that confocal microscopes can achieve a vertical nanometric resolution. For interferometric techniques, this is possible for all magnifications.

One also has to note that the values for the vertical resolution are given for flat measurements. For slopes, the ratio signal-to-noise decreases meaning a decrease of the vertical resolution and more difficulty to measure small height differences. Measuring the roughness of steep surfaces is thus also more difficult for optical profilers.

Contact probes can also be used to measure surface roughness. However, the tip acts as a low-pass filter and, consequently, the tip radius must be small enough to access high spatial frequencies. Typically, mechanical styluses have a tip whose radius is $>2 \mu \mathrm{m}$, thus preventing a complete collection of the surface roughness information. As an alternative, atomic force microscopes (AFMs) that have smaller tips, can be used. Different works report on the microlens surface measurement by AFM and a comparison of the different techniques can be found in Refs. 40 and 41 (Macro and Micro-Tribology and Mechanics of Magnetic Storage Systems).

It has to be noted that microlenses manufactured by photoresist reflow with subsequent $\mathrm{RIE}^{42}$ have usually a roughness with a random shape and with an amplitude $S_{q} \approx 1 \mathrm{~nm}$, see Fig. 2(a), which is optically good enough. On the other hand, microlenses that are manufactured based on micro-machining mastering ${ }^{43-45}$ have typically a roughness with a specific shape caused by the tool, see Fig. 2(b). In this case, more care should be taken to specify the roughness tolerance as the amplitude might significantly degrade the optical performance.

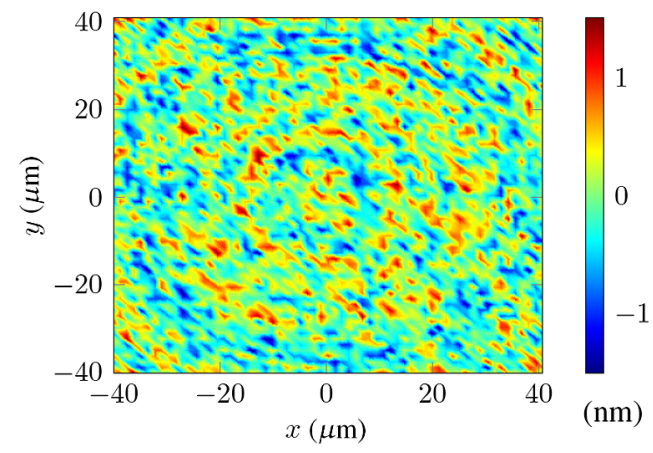

(a)

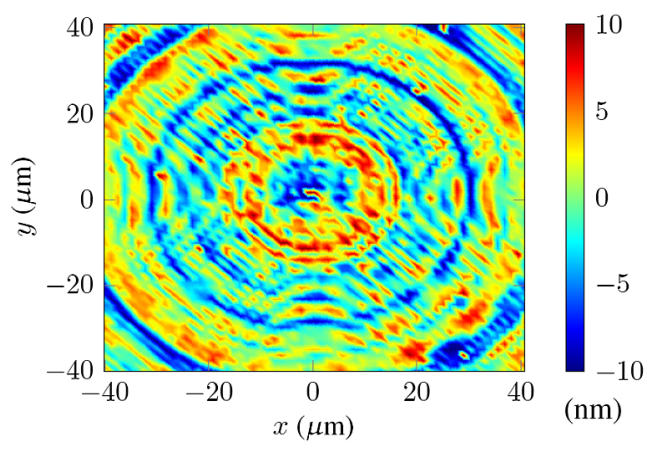

(b)

Fig. 2 Examples of roughness measurement by a CSI (Nexview from Zygo) equipped with a 100x microscope objective. Spatial sampling is $0.09 \mu \mathrm{m} /$ pixel and the spatial resolution is $0.34 \mu \mathrm{m}$. A high-pass spline filter whose cut-off period is $20 \mu \mathrm{m}$ is applied. (a) For a reflow-RIE microlens, the roughness is seen to be random. $S_{q}=0.5 \mathrm{~nm}$. (b) In the case of a micro-machined microlens, concentric circles created by the manufacturing tool are visible. $S_{q}=8.2 \mathrm{~nm}$. 


\section{Microlens Optical Functionality}

To ideally establish the performance of a microlens, the testing process should be performed under the exact same conditions as in the final application. Like this, the natural performance metric can be recorded and no testing uncertainty is introduced. For example, one should test a microlens by coupling a laser beam into an optical fiber, if it was designed for such a task. Then, the coupling efficiency directly gives the microlens performance.

However, from a practical point of view, performance testing under working conditions is rarely possible for different reasons: first, the optics manufacturer is generally in charge of the quality control and it cannot create a measurement setup for each type of microlens application. Moreover, an optical designer is most of the time not keen to share the full details of its optical system because of confidentiality.

For these reasons, the examination of the optical function of microlenses is usually reduced to standard tests. ${ }^{5,6}$ The most common quantity that is measured is the wavefront aberrations produced by the microlens. Considering this approach imply assuming a good correlation between the optical performance, the coupling efficiency in our previous example, and the wavefront aberration. Another common possibility is to measure the point spread function (PSF) of the microlens. One has to recall that these two types of measurements are performed in transmission. Both of them are discussed in this section.

\subsection{Wavefront}

The most common method for phase measurement is interferometry. Two types of interferometers have mainly been applied to microlens testing: the Mach-Zehnder type ${ }^{46-49}$ and the Twyman-Green type. ${ }^{37,47-49}$ Both interferometers are used in different configurations: usually, the probing wave is either plane or spherical, and the on-axis wavefront aberration is recorded.

Another technique that can be used to record the wavefront is using Shack-Hartman sensors. Those instruments typically consist of a 2D MLA placed in front of a camera and serve to sample the incoming wavefront. Each microlens produces a focal spot whose position in the image plane is a function of the incoming wavefront tilt at this particular microlens location. Thus, recording the focus position shift of all microlenses allows the reconstruction of the entire incoming wavefront.

With this technique, there is no need for multiple images recording contrary to interferometry, but it has the drawback that wavefront sampling is usually rather low. This technique is commercially available (e.g., Ref. 50).

\subsection{Point Spread Function}

The PSF of a microlens provides information about its performance. ${ }^{39,51}$ For microlenses used in imaging systems, the knowledge of the PSF leads to important information such as the resolution, the Strehl ratio, or the depth of field. This measurement is particularly important for high-quality imaging optics used in astronomical instruments. An example of the measurement of a microlens PSF is shown in Fig. 3.

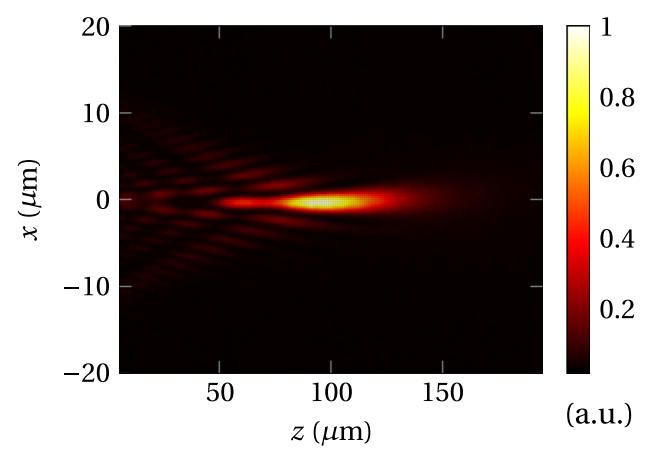

Fig. 3 Measured 3D PSF of a spherical microlens illuminated by an incident plane-wave. The effect of spherical aberration can be observed as the presence of many smaller intensity peaks before the main focal spot. 
This type of measurement is relatively straightforward to setup compared to interferometry as it consists mainly of a series of images taken with the help of a microscope objective at different locations along the optical axis and around the focal spot of the tested microlens. ${ }^{52}$ This operation is typically realized by mounting the microscope objective on a piezo actuator.

PSF measurements give a good insight into the optical performance of high-quality microlenses. Another advantage is the concentration of the focal spot on a very small area $(<10 \times 10 \mu \mathrm{m})$ allowing the use of high magnification and high NA microscope objectives. This condition is important since the NA of the microscope objective must be larger than the NA of the tested lens to resolve correctly the PSF and not omit its fine structure.

\subsection{Discussion}

The advantage of functional testing is a direct assessment of the optical performance provided the test is performed under valid conditions. The drawbacks of this approach are the cost to setup these valid conditions and the difficulty to make a link with the surface.

Valid conditions mean that the testing conditions must be equivalent to the working conditions. In particular, the test illumination must be similar to the working one, in terms of wavelength, wavefront, and irradiance. Unless it has a very low NA, the surface of a microlens is generally aspheric to reduce the aberrations. As the asphericity depends on a specific illumination, using a different illumination will result in artificial aberrations. This means that the standard plane-wave and spherical wave illuminations are not sufficient to test aspheres. This poses also difficulties when the material is non-transparent to visible light, e.g., silicon. All this limits thus the possible implementation of such a method in an industrial environment where dozens of different microlenses are processed and tested in parallel.

Another drawback of these measurements is the accuracy assessment. As these types of characterization take into account not only the microlens geometrical parameters but also the physical ones, one could think that the performance evaluation would be more accurate in comparison with surface measurements. However, one also has to take into consideration the error caused by the optical setup. Indeed, relay optics between the camera and the microlens add errors. In particular, a microscope objective is required to expand the field-of-view (FOV) and additional aberrations are induced. This aspect is discussed more deeply in Sec. 5.2. In summary, we can say that functional testing is less practical to setup than surface measurement.

\section{Microlens Arrays}

MLAs typically consist of the repetition of a given microlens design arranged on a specific lattice. Figure 4 shows two examples of MLAs, one with spherical microlenses and one with cylindrical microlenses. Typical applications of such arrays are beam homogenizers ${ }^{53}$ or micro-optical projectors. ${ }^{54}$ The design of an MLA requires the definition of the lattice, meaning defining a pitch value, and an arrangement (square and hexagonal). This generalization from a single microlens to an array changes the approach of the characterization, and consequently, of metrology.

On the one hand, assessing the optical performance of an MLA is more complex compared to single microlenses. Indeed, for most applications, the optical function of an MLA is given by the

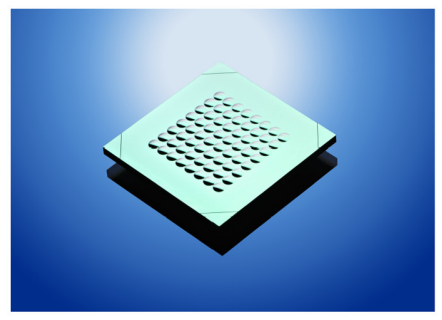

(a)

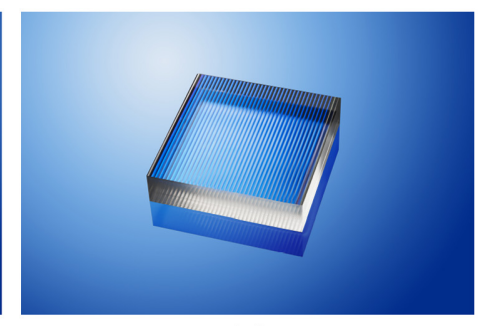

(b)

Fig. 4 Examples of MLAs in different materials and with different geometries. (a) Spherical microlenses made in silicon. (b) Cylindrical microlenses made in fused silica. 


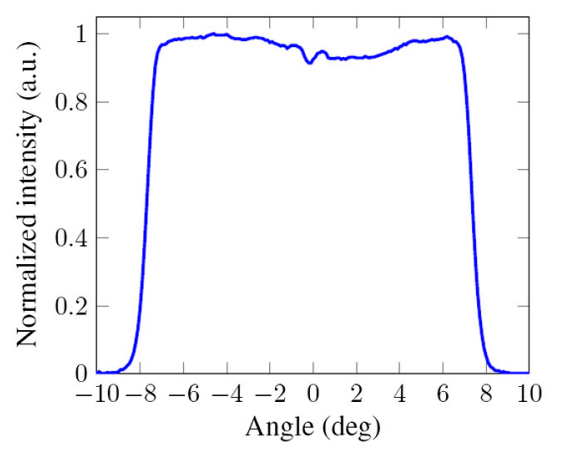

(a)

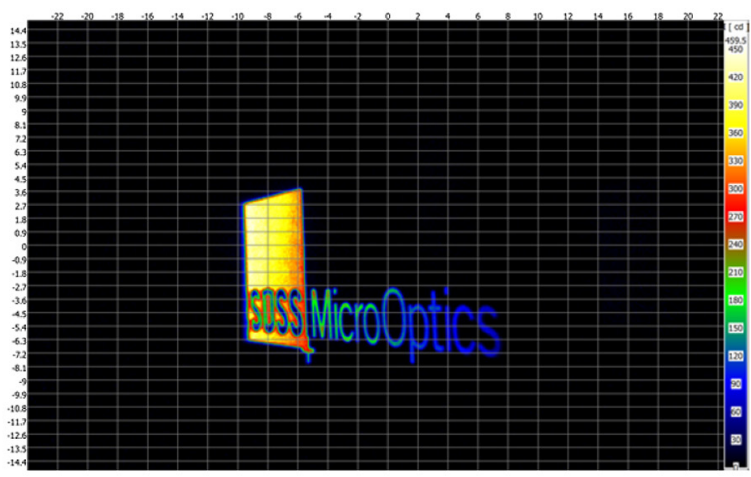

(b)

Fig. 5 Functional test examples: (a) Measured angular spectrum of a beam-homogenizer consisting of a double-sided MLA with 200 cylindrical microlenses on each side. (b) Recorded projection (luminous intensity) by a micro-optical projector consisting of a double-sided MLA with 256 channels and microlenses on each side.

combination of the optical function of the individual microlenses. One exception is MLAs used for Shack-Hartman sensors as each microlens is an independent imaging channel, meaning each microlens produces a different image, and no recombination effects need to be considered.

When the MLA functionality combines multiple microlenses, one ends up with a very complicated function that is in practice highly difficult to establish from the measurement of the individual geometrical parameters. A functional test of the entire MLA is thus required to ensure a proper assessment of the optical performance. As an example, a beam homogenizer is usually tested by the measurement of its angular spectrum, see Fig. 5(a). This measurement is realized by recording the intensity with a goniometric system far from the MLA, which is illuminated by a plane-wave. Likewise, the quality of a micro-projector is evaluated by processing the projected image, Fig. 5(b). This measurement is achieved by illuminating the micro-projector (e.g., with a light-emitting diode) and by recording the projected luminous intensity on a screen. A Fourier lens placed between the micro-projector and the screen provides the conversion between the spatial and angular space.

On the other hand, the MLA metrology for fabrication process improvement is more similar to the single microlens approach. Indeed, the only additional geometrical parameter that has to be controlled is the pitch between the microlenses or in other words, their position. For instance, this can be realized by capturing in the same surface measurement two or more microlenses by an optical or mechanical profiler. It is important to perform such a test, as we have observed pitch variations at the edge of MLAs, where the lattice symmetry is broken. ${ }^{55}$

As a side note, it has to be mentioned that in the case of MLAs, process optimization goes also through the increase of uniformity within the MLA. In particular, spatial variations of the fabrication process lead to different ROCs, thus different focal lengths. For this reason, assessing this non-uniformity is necessary to reduce it. ${ }^{56}$

In conclusion, for MLAs contrarily to single microlenses, it is no more possible to have a single surface measurement that provides both performance assessment and feedback for process optimization. As a consequence, MLAs metrology requires at least two types of measurements.

\section{Discussion}

So far, we have presented different methods to measure specific microlens features. In this section, we discuss their limitations and compare them. We also propose general guidelines to select the right approach depending on the purpose of the characterization.

\subsection{Geometrical Limitations}

Here, we discuss the influence of the microlens geometry, mainly its diameter, height, and maximum surface slope, on the measurement process. Indeed, these quantities are fundamental 
factors that limit the usability of the different measurement techniques. We have to stress that these geometrical limitations concern only surface profilers, both mechanical and optical, as functionality testing is performed under working conditions and thus does not face this problem.

For stylus tools, the diameter of a refractive microlens, typically $<1 \mathrm{~mm}$, and its height, usually below $300 \mu \mathrm{m}$, are not limiting factors. Indeed, profiles over several centimeters and with a vertical range over $1 \mathrm{~mm}$ can be recorded with standard commercial instruments. For surface slopes, the limiting factor lies in the geometry of the stylus tip, which is a cone ended by a portion of a ball. Indeed, the maximum surface angle of the rounded part of the tip must be higher than the slope of the measured surface to have proper contact. A common value for the maximum slope of the tip is $60 \mathrm{deg}$, but tips exist with higher values. This value is sufficient for almost all microlenses used in real systems nowadays. As a consequence, the microlens geometry is not a concern for stylus instruments.

For optical surface profilers working in reflection, the story is completely different. Indeed, the geometrical limitations depend almost entirely on the imaging system, and especially on its central compound, the microscope objective. Indeed, its FOV gives the largest measurable microlens diameter, its working distance (WD) gives the maximum measurable height, and its NA gives the limit of the maximum surface slope that can be measured using specular reflection, see Fig. 6(a). As shown previously, the microlens surfaces are very smooth, and going above this limit by using scattered light seems to us almost unfeasible even if some work is currently done ${ }^{57,58} \mathrm{We}$ have thus to look at the microscope objectives properties that are used in those optical surface profilers to estimate the limitations.

The number of available microscope objectives is relatively limited. Here, we briefly compare the microscope objectives that equip selected commercial optical profilers: the confocal microscope $\mu$ surf from Nanofocus, the CSI Nexview from Zygo, and the combined instrument (confocal + CSI) S-neox from Sensofar. ${ }^{59-61}$ For the $10 \times$ magnification, the NA is similar for all objectives. For the $20 \times$, interferometric objectives have a NA of 0.4 . For confocal, objectives with an NA of up to 0.6 can be obtained. This suggests an advantage of confocal microscopes over CSIs. This is also confirmed for $50 \times$ and $100 \times$ objectives. For instance, the $50 \times$ Mirau objective has a NA of 0.55 , whereas confocal microscopes can be equipped with objectives whose NA is 0.8 .

These considerations are summarized in Fig. 6(b), which presents the estimated maximum measurable slope as a function of the FOV. Due to non-interferometric microscope objectives, confocal microscopes have a better capability to measure surface slopes. It has to be stressed that the given values represent only an estimation. Other factors, such as the sampling in the case of interferometric measurements, or the signal-to-noise ratio are other important limiting factors.

To put these considerations in a more practical context, we use three instruments based on different techniques to measure the surface of a 2-mm diameter reference ball. First, we use the CSI Nexview from Zygo equipped with a $100 \times$ NA 0.85 WD $0.5-\mathrm{mm}$ microscope objective. Because the FOV is reduced for high magnifications, we also use stitching with this instrument.

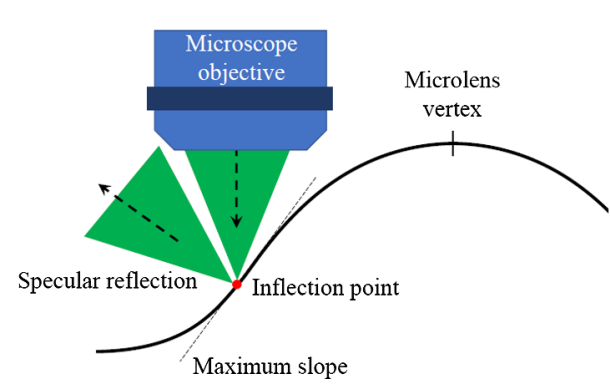

(a)

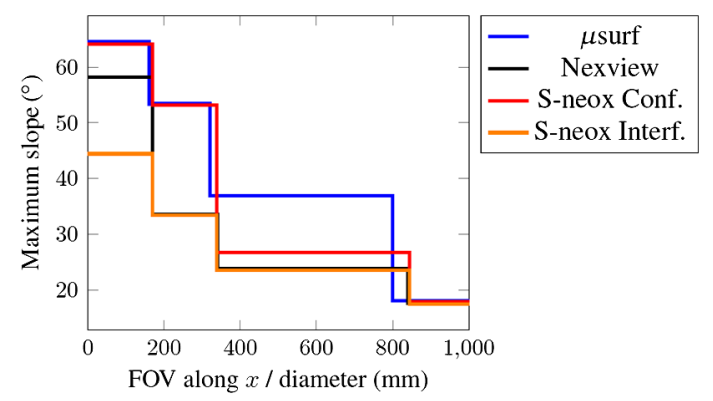

(b)

Fig. 6 (a) Illustration (not to scale) of the maximum surface slope of a reflow-RIE based microlens. In this example, the NA of the microscope objective is not sufficient to collect the light that is reflected at the steepest point of the surface. (b) Comparison between different optical surface profilers in terms of surface geometry limitation. Confocal microscopes present an advantage over CSIs for FOV below $800 \mu \mathrm{m}$ as the maximum measurable slope is higher. 


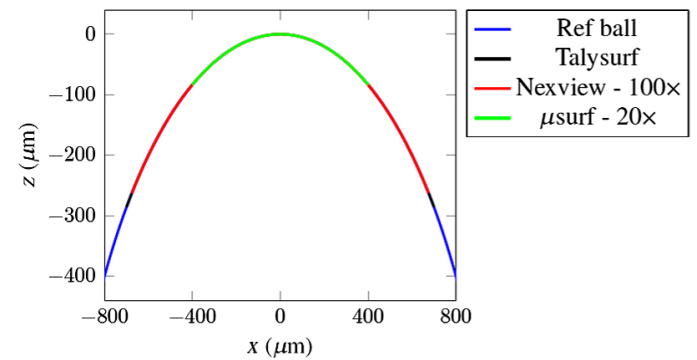

(a)

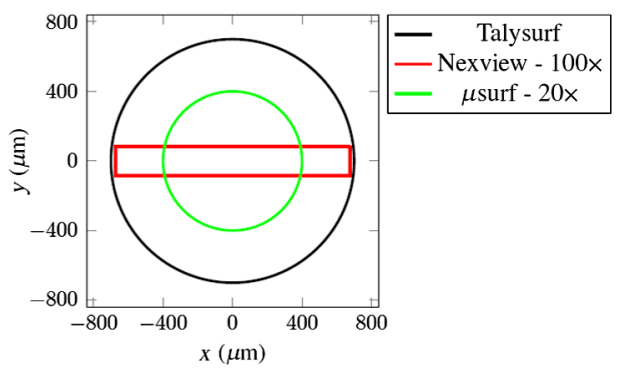

(b)

Fig. 7 Surface measurements of a 1-mm ROC reference ball by different measuring instruments. (a) Profiles. (b) Top view: FOV. The stylus tool allows gathering more information about the surface.

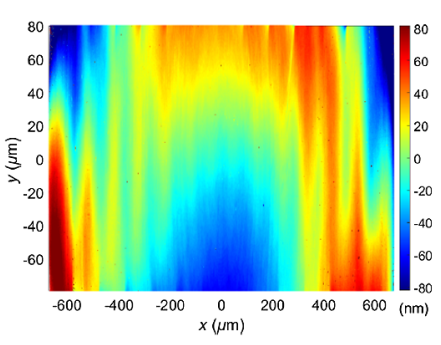

(a)

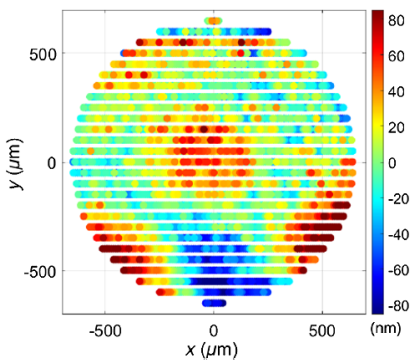

(b)

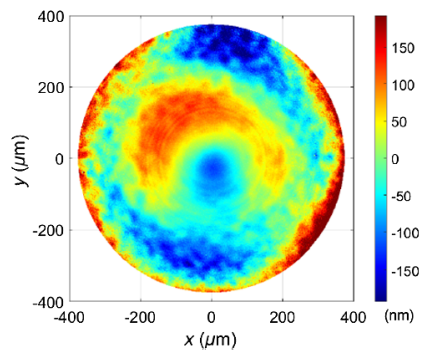

(c)

Fig. 8 Form of the best-sphere fit residual for the three instruments calibrated following the manufacturer guidelines. (a) Nexview $100 \times, \mathrm{RMS}=34 \mathrm{~nm}$, sampling $x: 0.17 \mu \mathrm{m}, y: 0.17 \mu \mathrm{m}$. (b) Talysurf, RMS $=55 \mathrm{~nm}$, sampling $x: 10 \mu \mathrm{m}, y: 40 \mu \mathrm{m}$. (c) $\mu$ surf $20 \times$, RMS $=78 \mathrm{~nm}$, sampling $x$ : $1.6 \mu \mathrm{m}, y: 1.6 \mu \mathrm{m}$. As the ball surface has a deviation from a spherical surface below $10 \mathrm{~nm}$ RMS, these residuals are considered measurement artifacts. In particular, the vertical lines visible in panel (a) are the consequence of the stitching operation (it is worth noting that the $x$ and $y$ scales are different).

Second, we use the stylus Talysurf from Taylor Hobson, which can record 3D surface information and not only surface profiles. Finally, we use the confocal microscope $\mu$ surf custom from Nanofocus equipped with a $20 \times$ NA 0.6 WD $0.9-\mathrm{mm}$ microscope objective. Stitching is not used in this case.

Figure 7 shows the reference ball surface information that can be collected by these three instruments. As expected, the stylus probes the largest surface area for a comparable time frame but with the drawback of low sampling, which prevents the acquisition of the high spatial frequency information of the surface.

To evaluate the quality of these measurements, the best-sphere fit residuals (irregularities) are shown in Fig. 8. All three measurements present artifacts when used in the manufacturer configuration. For the confocal measurement, it has been shown that this error can be corrected. ${ }^{36}$

This comparison is summarized in Table 1. Information about measurement time is also included. Like this, any user can select the measuring system based on his needs in terms of surface information, quality of the measurement, and measurement time.

In addition to their relative difficulty to measure steep slopes, one disadvantage of interferometric systems is that another type of errors occurs during the phase reconstruction, which causes a localized artificial high roughness. This is shown in Fig. 9(a), which presents the measured irregularity (best asphere fit residual) of an aspheric surface. Even though the exact origin of this issue might be difficult to determine, this problem clearly limits the capability of the instrument. However, this error can be well corrected when the surface is smooth ${ }^{55}$ as it is shown in Fig. 9(b), which presents the same exact measurement after being processed.

This discussion would not be complete without a comment on the range of geometries spanned by real microlenses. First, we can say from our experience that a large majority of the 
Table 1 Comparison between three different measurement methods using three different instruments. The amount of information that is collected (surface area) and its quality (RMS value) are different from one method to the other, but the measurement time as well. A trade-off between these different parameters has to be found.

\begin{tabular}{lccccc}
\hline \hline Instrument & $\begin{array}{c}\text { Measured dim. } \\
(\mu \mathrm{m})\end{array}$ & $\begin{array}{c}\text { Max slope } \\
(\mathrm{deg})\end{array}$ & $\begin{array}{c}\text { ROC } \\
(\mu \mathrm{m})\end{array}$ & $\begin{array}{c}\text { RMS } \\
(\mathrm{nm})\end{array}$ & $\begin{array}{c}\text { Time } \\
(\mathrm{min})\end{array}$ \\
\hline Nexview 100x & Rect. 1350 $\times 160$ & 43 & 1001.2 & 34 & 5 \\
Talysurf & Diam. 1400 & 45 & 1000.1 & 55 & 6.3 \\
$\mu$ surf custom 20x & Diam. 750 & 23 & 1001.2 & 78 & 0.25 \\
\hline \hline
\end{tabular}

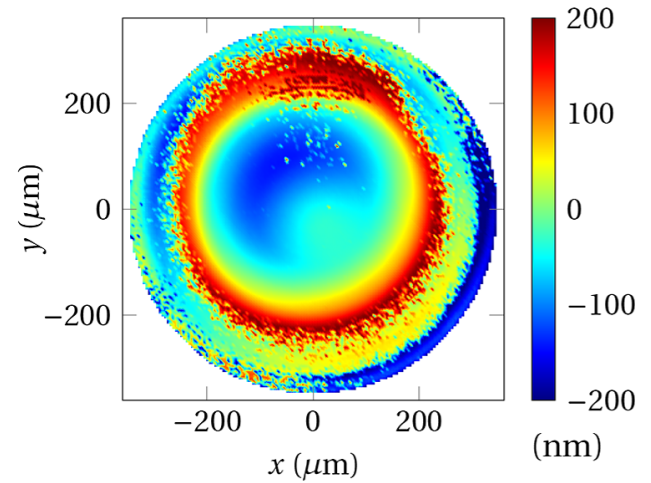

(a)

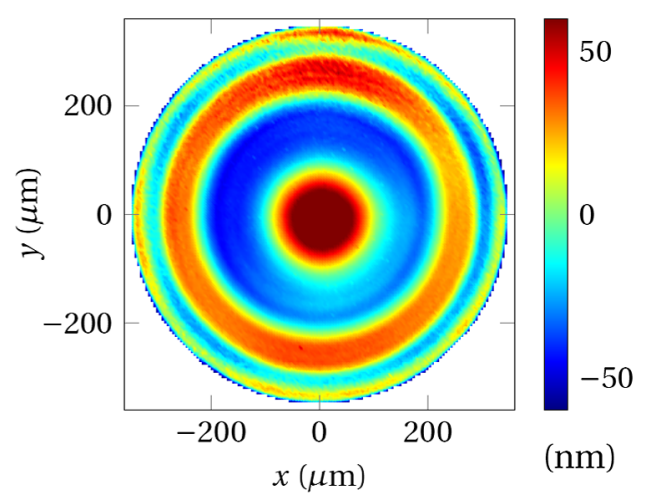

(b)

Fig. 9 (a) The measured irregularity of an aspheric microlens $(R=460 \mu \mathrm{m}, \kappa=-4)$ puts in evidence artifacts in CSI measurements due to the bad reconstruction of the surface from phase information. (b) The same measurement after correction.

microlenses fabricated by resist reflow with a subsequent RIE process are measurable by optical surface profilers without stitching. However, the more recent fabrication technique based on polymer replication with direct writing molds, which allows creating steeper and higher microlenses, represents a real challenge for surface metrology. Currently, the only available option for this technology is to use mechanical styluses that perform multiple scans to obtain the full 3D surface information. As mentioned previously, the main drawbacks of this approach are the measurement time and the difficulty to measure sticky surfaces (e.g., polydimethylsiloxane).

\subsection{Accuracy and Precision}

Measurement accuracy and precision ${ }^{62}$ are fundamental limits and determine the highest microlens quality that can be achieved as these limits restrain the feedback information needed to optimize the fabrication process. On the one hand, it is easy to assess precision by running multiple measurements under repeatable or reproducible conditions. Then, a comparison with the desired tolerance allows us to decide whether the instrument is capable to resolve what we are looking for. Figure 10 shows the ROC precision under repeatable conditions for two different instruments for the same 2-mm diameter reference ball. The ROC precision mainly depends on the quality of the actuators in the systems as well as on the vibrations present during the measurement.

On the other hand, accuracy assessment for any measured parameter is trickier as most of the measuring systems are complex and a model for the measurement process is not available. A simple workaround is to estimate the measurement accuracy using a reference object or calibration artifact. This discussion about accuracy does not concern functional testing in real working conditions. 


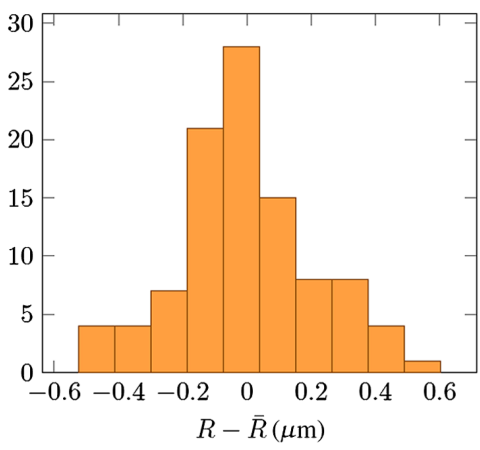

(a)

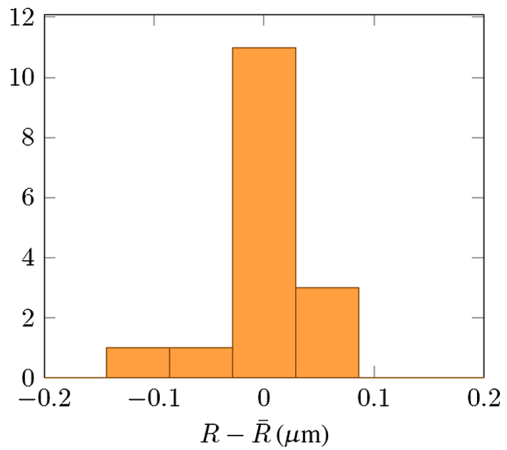

(b)

Fig. 10 Measured ROC distribution under repeatable conditions with two different tools. (a) Nexview: 100 occurences, $\sigma=0.2 \mu \mathrm{m}$. (b) Talysurf: 15 occurences, $\sigma=0.05 \mu \mathrm{m}$. The ROC nominal value is $1000 \mu \mathrm{m}$.

Several calibration artifacts for surface measurements exist. First, flat reference mirrors are used to correct the residual flatness of optical surface profilers. However, these references are of limited use for microlenses, whose surface largely deviates from a plane. More interesting are the reference balls of very high quality that are commercially available (e.g., Ref. 63). A random ball test ${ }^{64}$ performed with those balls provides a reference surface with arbitrary accuracy. ${ }^{55}$ Thereby, accuracy can be assessed and measurement error corrected. The limitation of this approach is the limited number of available ball ROCs. Also, this approach is of limited usefulness for aspheric microlenses.

For the functional tests performed in transmission, i.e., wavefront of PSF measurements, which require relay optics between the tested microlens and the camera, the situation is even more tricky. Indeed, no artifact exists for transmission, meaning that one cannot evaluate easily the accuracy. In macro-optics, one option is to use computer-generated holograms. This option is not applicable to micro-optics for scale reasons, with the exception of cylindrical microlenses. ${ }^{65}$

This issue leaves us with the assumption that the error caused by the relay optics is negligible compared to the aberrations provoked by the tested microlens. This is however a strong assumption that is likely not valid in practice. An alternative would be to evaluate the error based on ray-tracing and perfect knowledge of the relay optics, which is not easy to do. This difficulty to evaluate the measurement uncertainty leaves us in the dark and is a real drawback to increase the microlens quality. We believe this is one of the main drawbacks of testing in transmission.

\subsection{Industrial Perspective}

In an industrial manufacturing environment, microlens surface measurements are preferred over functional testing (wavefront, PSF) for multiple reasons: first, because the surface must be known to develop, optimize and control the fabrication process. Second, microlenses with different materials, working at different wavelengths, having different functionalities thus different surface forms are manufactured. This would require many different optical setups to test the optical function of all these microlenses, whereas a single optical surface profiler can handle most of them.

Second, as the surface profilers have initially been developed for the semiconductor industry, many suppliers exist that propose instruments designed for wafer-level inspection. Most of the techniques can be commercially found. ${ }^{66-72}$ On the other hand and to our knowledge, there is no commercially available instruments that measure the microlens functionality in transmission and that are equipped for wafer-level optics. The fact that building in-house an instrument that can handle wafer-level micro-optics is extremely complicated also pushes microlens manufacturers to use already existing surface profilers.

\subsection{Guidelines}

Based on the previous discussions, we propose some guidelines about the practice of microlens metrology. First, we discuss what are the steps that must be undertaken to obtain information for 
fabrication process optimization. In a second time, we discuss the evaluation of the microlens performance.

\subsubsection{Feedback for fabrication process optimization}

As previously mentioned, the knowledge of the surface is the piece of information required to optimize the fabrication process, and in this case, single microlenses or MLAs are treated equivalently. As long as the microlens surface can be measured in reflection by optical surface profilers, the characterization is relatively straightforward. The interesting question is how should we proceed when the surface slope is too steep for this approach to be applied?

If a single profile is sufficient, due to an assumed rotational symmetry, or if the microlens is cylindrical, then stylus measurements are likely to be the best option. If the full surface is necessary, then it depends because there is not a simple technique to achieve this task. Maybe it is sufficient to use a higher NA microscope objective with a reduced FOV that cannot encompass entirely the microlens and to stitch several frames together. This might, however, take a very long time or be limited by computing resources. Also, care should be taken concerning artifacts introduced during the stitching process. The other option is to combine measured $2 \mathrm{D}$ profiles measured by stylus to obtain the 3D surface. This might be the best alternative as this technique has no geometrical limitations as discussed previously. Unfortunately, the measurement time becomes larger, but no convincing alternative exists yet.

\subsubsection{Determination of the optical performance}

Evaluation of the optical performance can be obtained by direct measurements or derived from geometrical and physical parameters measurements. In the latest case, the approach depends on the measured object. Here, we differentiate between simple and complex micro-optical systems.

If this object is a single microlens, a small array, or an MLA with independent channels, meaning that the performance is almost entirely determined by the surface, then performance evaluation based on the surface measurement is an advantageous approach for two reasons: first, as seen previously, surface measurement is easy, accurate, and automated instruments are available. Second, the surface has to be measured in any case to control and optimize the fabrication process.

Even if this approach is quite simple from a measurement point of view, the challenge becomes to assess the performance based on the surface information. This is usually performed by assigning tolerances to the surface. Eventually, the performance can also be derived through ray-tracing or other forms of optical simulation.

This approach consists of measuring the surface and deriving the optical performance, which offers more flexibility than direct functional measurement, as surface measurements do not depend on the microlens material, the operating wavelength, and the nominal surface form (spherical, aspheric, and cylindrical). This is likely the main reason that explains why it is the most used approach for simple micro-optical systems.

By complex micro-optical systems, we mean large MLAs whose optical channels are combined. In this case, as mentioned previously, it is very difficult, if not impossible, to predict the optical performance with sufficient accuracy based on the geometrical parameters. Indeed, some of them might not even be measurable. In this case, there is no other option than performing a functional test specific to the micro-optical system.

\section{Conclusion}

Microlens metrology gathers measurement techniques that are used to obtain appropriate information to, first, optimize the microlens fabrication process and to, second, assess the microlens performance.

Measuring the form of the microlens surface is of uttermost importance as this piece of information provides the necessary feedback to optimize the fabrication process. Moreover, for single microlenses, the surface also defines the optical performance. Microlens metrology is thus 
closely related to surface metrology and uses instruments developed in this field. A smart choice of surface profilers depends on the microlens geometry. Indeed, optical surface profilers have difficulty measuring steep and large microlenses, whereas contact probes might have issues with sticky surfaces. For this reason, to span the full range of possible microlens geometries, several instruments based on different techniques are required.

As an alternative, the optical performance can also be assessed by a functional test. An example is the common wavefront aberration measurement by interferometry. To be meaningful, these tests must be performed under working conditions, which is impractical in an industrial environment. Nevertheless, for complex micro-optical systems such as large MLAs, the optical performance cannot be simply assessed through surface measurements and dedicated optical testing setups must be used.

In conclusion, microlens metrology is not a standard industrialized process yet and is still under development. As the micro-optics technology has gained importance and is evolving rapidly, we expect that new metrological instruments with improved capability, especially in terms of automation, time, and accuracy, will be available. However, we fear that no revolution in the different measurement principles will be foreseen in the near future. One also hopes that microoptics will benefit from the development of other technologies such as additive manufacturing, which represents a tremendous challenge for metrology.

\section{Acknowledgments}

We thank our colleagues from the Metrology and R\&D departments for useful discussions and their help toward this publication. The authors declare no conflict of interest.

\section{References}

1. C. A. Edwards, H. M. Presby, and C. Dragone, "Ideal microlenses for laser to fiber coupling," J. Lightwave Technol. 11, 252-257 (1993).

2. T. E. L. Fred and M. Dickey, Laser Beam Shaping Applications, CRC Press, Boca Raton, Florida (2017).

3. M. Sieler et al., "Microoptical array projectors for free-form screen applications," Opt. Express 21, 28702-28709 (2013).

4. "Optics and photonics—microlens array—part 1: vocabulary," ISO:14880-1:2019, International Organization for Standardization (2019).

5. "Optics and Photonics-microlens array-part 2: test methods for wavefront aberrations," ISO:14880-2:2006, International Organization for Standardization (2006).

6. "Optics and photonics-microlens array-part 3: test methods for optical properties other than wavefront aberrations," ISO:14880-3:2006, International Organization for Standardization (2006).

7. "Optics and photonics - microlens array - part 2: test methods for geometrical properties," ISO:14880-4:2006, International Organization for Standardization (2006).

8. "Optics and photonics—microlens array—part 5: guidance on testing," ISO:14880-5:2010, International Organization for Standardization (2010).

9. R. Voelkel, M. Eisner, and K. J. Weible, "Fabrication of aspherical microlenses in fused silica and silicon," Proc. SPIE 4440, 40-43 (2001).

10. L. Li and A. Y. Yi, "Design and fabrication of a freeform microlens array for a compact large-field-of-view compound-eye camera," Appl. Opt. 51, 1843-1852 (2012).

11. R. Guo et al., "Micro lens fabrication by means of femtosecond two photon photopolymerization," Opt. Express 14, 810-816 (2006).

12. C. H. Lin et al., "Fabrication of microlens arrays in photosensitive glass by femtosecond laser direct writing," Appl. Phys. A 97, 751-757 (2009).

13. H. Schmitt et al., "Full wafer microlens replication by UV imprint lithography," Microelectron. Eng. 87(5), 1074-1076 (2010).

14. T. Miyashita, "Standardization for microlenses and microlens arrays," Jpn. J. Appl. Phys. 46, 5391-5396 (2007). 
15. T. Hou et al., "Fabrication, characterization, and applications of microlenses," Appl. Opt. 54, 7366-7376 (2015).

16. W. Yuan et al., "Fabrication of microlens array and its application: a review," Chin. J. Mech. Eng. 31, 16 (2018).

17. R. Voelkel, "Wafer-scale micro-optics fabrication," Adv. Opt. Technol. 1(3), 135-150 (2012).

18. J. Schwider et al., "Homogeneity testing by phase sampling interferometry," Appl. Opt. 24, 3059-3061 (1985).

19. Schott, "Homogeneity of optical glass," https:/www.schott.com/d/advanced_optics/ 8085e554-e0a3-47f5-a5fa-af72b4ff8955/1.2/schott_tie_26_homogeneity_of_optical_glass_ eng.pdf (accessed 29 March 2021).

20. "Optics and photonics_-preparation of drawings for optical elements and systems_part 18: stress birefringence, bubbles and inclusions, homogeneity, and striae,” ISO 10110-18:2018, International Organization for Standardization (2018).

21. "Optics and photonics—-specification of raw optical glass," ISO 12123:2018, International Organization for Standardization (2018).

22. R. J. Noll, "Effect of mid- and high-spatial frequencies on optical performance," Opt. Eng. 18(2), 137-142 (1979).

23. H. Gross, B. Dörband, and H. Müller, Testing the Geometry of Optical Components, Chapter 53, pp. 679-783, John Wiley \& Sons, Ltd. (2012).

24. J. E. Harvey, J. D. Lytle, and H. E. Morrow, "Light scattering characteristics of optical surfaces," Proc. SPIE 0107, 41-47 (1997).

25. E. L. Church, H. A. Jenkinson, and J. M. Zavada, "Relationship between surface scattering and microtopographic features," Opt. Eng. 18(2), 125-136 (1979).

26. S. Schröder et al., "Modeling of light scattering in different regimes of surface roughness," Opt. Express 19, 9820-9835 (2011).

27. D. M. Aikens, J. E. DeGroote, and R. N. Youngworth, "Specification control of mid-spatial frequency wavefront errors optical systems," Front. Opt./Laser Sci. XXIV/Plasmonics and Metamater./Opt. Fabrication and Testing, OTuA1, Optical Society of America (2008).

28. J. Goodman, Introduction to Fourier Optics, Roberts \& Company Publishers, Englewood, Colorado (2005).

29. "Optics and photonics - preparation of drawings for optical elements and systems - part 5: surface form tolerances," ISO 10110-5:2015, International Organization for Standardization (2015).

30. R. Artigas, Imaging Confocal Microscopy, pp. 237-286, Springer, Berlin, Heidelberg (2011).

31. P. de Groot, Coherence Scanning Interferometry, pp. 187-208, Springer, Berlin, Heidelberg (2011).

32. T. Colomb and J. Kühn, Digital Holographic Microscopy, pp. 209-235, Springer, Berlin, Heidelberg (2011).

33. P. Lehmann, "Vertical scanning white-light interference microscopy on curved microstructures," Opt. Lett. 35, 1768-1770 (2010).

34. M. Rahlves, B. Roth, and E. Reithmeier, "Systematic errors on curved microstructures caused by aberrations in confocal surface metrology," Opt. Express 23, 9640-9648 (2015).

35. R. Su et al., "On tilt and curvature dependent errors and the calibration of coherence scanning interferometry," Opt. Express 25, 3297-3310 (2017).

36. J. Béguelin et al., "Correction of spherical surface measurements by confocal microscopy," Meas. Sci. Technol. 31, 075002 (2020).

37. J. Schwider and O. R. Falkenstoerfer, "Twyman-Green interferometer for testing microspheres," Opt. Eng. 34(10), 2972-2975 (1995).

38. R. E. Reason, M. Hopkins, and R. I. Garrod, Report on the Measurement of Surface Finish by Stylus Methods, Taylor \& Hobson (1946).

39. M. Baranski et al., "A simple method for quality evaluation of micro-optical components based on 3d ipsf measurement," Opt. Express 22, 13202-13212 (2014).

40. C. Y. Poon and B. Bhushan, "Comparison of surface roughness measurements by stylus profiler, AFM and non-contact optical profiler," Wear 190(1), 76-88 (1995). 
41. A. Duparré et al., "Surface characterization techniques for determining the root-meansquare roughness and power spectral densities of optical components," Appl. Opt. 41, 154-171 (2002).

42. P. Nussbaum et al., "Design, fabrication and testing of microlens arrays for sensors and microsystems," Pure Appl. Opt. 6, 617-636 (1997).

43. N. C. R. Holme, T. W. Berg, and P. G. Dinesen, "Diamond micro-milling for array mastering," Proc. SPIE 7062, 70620J (2008).

44. G. E. Davis, J. W. Roblee, and A. R. Hedges, "Comparison of freeform manufacturing techniques in the production of monolithic lens arrays," Proc. SPIE 7426, 742605 (2009).

45. M. Wielandts and R. Wielandts, "Novel method for fabrication of monolithic multi-cavity molds and wafer optics," Proc. SPIE 9633, 96331K (2015).

46. S. Haselbeck et al., "Microlenses fabricated by melting a photoresist on a base layer," Opt. Eng. 32(6), 1322-1324 (1993).

47. K. O. Mersereau et al., "Testing and measurement of microlenses," Proc. SPIE 1992, 210-215 (1993).

48. S. Reichelt and H. Zappe, "Combined Twyman-Green and Mach-Zehnder interferometer for microlens testing," Appl. Opt. 44, 5786-5792 (2005).

49. V. Gomez et al., "Micro-optic reflection and transmission interferometer for complete microlens characterization," Meas. Sci. Technol. 20, 025901 (2009).

50. Optocraft GmbH, "SHS Inspect," https://www.optocraft.de/fileadmin/Download/OPC_ Productfolder_SHSInspect_E_Web_170615.pdf (accessed 29 March 2021).

51. M.-S. Kim, T. Scharf, and H. P. Herzig, "Small-size microlens characterization by multiwavelength high-resolution interference microscopy," Opt. Express 18, 14319-14329 (2010).

52. J. Béguelin et al., "Optical characterization of high numerical aperture microlenses for quality assessment and fabrication process optimization," Appl. Opt. 59, 3601-3607 (2020).

53. R. Voelkel and K. J. Weible, "Laser beam homogenizing: limitations and constraints," Proc. SPIE 7102, 71020J (2008).

54. M. Sieler et al., "Ultraslim fixed pattern projectors with inherent homogenization of illumination," Appl. Opt. 51, 64-74 (2012).

55. J. Béguelin, "On the assessment of aspheric refractive microlenses," p. 143 (2020).

56. R. Kirner et al., "Improvements on the uniformity of large-area microlens arrays in fused silica," Opt. Express 27, 6249-6258 (2019).

57. M. F. Fay, X. C. de Lega, and P. de Groot, "Measuring high-slope and super-smooth optics with high-dynamic-range coherence scanning interferometry," in Classical Opt., OW1B.3, Optical Society of America (2014).

58. M. Thomas et al., "Optical topography measurement of steeply-sloped surfaces beyond the specular numerical aperture limit," Proc. SPIE 11352, 1135207 (2020).

59. Zygo Corporation, "Profiler Objectives for Zygo 3D optical surface profilers," https:// www.zygo.com/products/metrology-systems/3d-optical-profilers/nexview-nx2/ (accessed 29 March 2021).

60. Sensofar, "S neox 3D optical profiler-Specifications," https://www.sensofar.com/metrology/ products/sneox/specifications/ (accessed 29 March 2021).

61. Olympus, "Objective specifications," https://www.olympus-ims.com/en/component/objectives/ (accessed 29 March 2021).

62. "Evaluation of measurement data—guide to the expression of uncertainty in measurement," standard, Joint Committee for Guides in Metrology (2008).

63. Saphirwerk, "Precision balls—of the highest grade," https://saphirwerk.com/assets/ Downloads/Saphirwerk-precision-balls.pdf (accessed 29 March 2021).

64. Y. Zhou et al., "Application of the random ball test for calibrating slope-dependent errors in profilometry measurements," Appl. Opt. 52, 5925-5931 (2013).

65. J. Lamprecht, N. Lindlein, and J. Schwider, "Null test measurement of high-numericalaperture cylindrical microlenses in transmitted light," Proc. SPIE 5180, 253-260 (2003).

66. Sensofar, "S neox 3D optical profiler," https://www.sensofar.com/wp-content/uploads/2019/ 03/BR90-05D-EN.pdf (accessed 29 March 2021). 
67. NanoFocus AG, " $\mu$ surf custom," https://www.nanofocus.com/products/usurf/usurf-custom (accessed 29 March 2021).

68. Mahr GmbH, "MarSurf CM select," https://metrology.mahr.com/en-int/products/article/ 6350006-flaechenhafte-3d-messung-marsurf-cm-select/ (accessed 29 March 2021).

69. Bruker, "Contour Elite X 3D Optical Profiler," https://www.bruker.com/products/surfaceand-dimensional-analysis/3d-optical-microscopes/3d-surface-metrology/contour-elite-x/ overview.html (accessed 29 March 2021).

70. Zygo Corporation, "Nexview NX2," https://www.zygo.com/products/metrology-systems/ 3d-optical-profilers/nexview-nx2/ (accessed 29 March 2021).

71. Lyncée Tec SA, "DHM - R series," https://www.lynceetec.com/wp-content/uploads/2014/ 02/LynceeTec_DHM_Rseries_Datasheet-2020.pdf (accessed 29 March 2021).

72. Taylor Hobson, "Form Talysurf PGI Matrix," https://www.taylor-hobson.com/products/ surface-profilers/optics-pgi/pgi-matrix (accessed 29 March 2021).

Jeremy Béguelin received his MSc degree in physics and his PhD in photonics from the École Polytechnique Fédérale de Lausanne (EPFL) in 2016 and 2020, respectively. For his master thesis, he worked on optical MEMS as a visiting scholar at UC Berkeley in the integrated photonics laboratory. His $\mathrm{PhD}$ thesis focused on refractive micro-optics and was realized in the industry at SUSS MicroOptics. In 2021, he joined L.E.S.S. SA, leading the optical development of the company.

Reinhard Voelkel received his diploma in physics in 1989 and his $\mathrm{PhD}$ in 1994 from the University of Erlangen-Nuremberg, Germany. He worked at the University of Neuchâtel, Switzerland. He is co-founder and CEO of SUSS MicroOptics SA, expert for Innosuisse - the Swiss Innovation Agency, member of the DGaO, SSOM, EOS, senior member of OSA, SPIE Fellow, and member of Sand Hill Angels. He is author or co-author of more than 200 scientific publications.

Toralf Scharf has a background in surface physics (MSc) and physical chemistry ( $\mathrm{PhD})$. He is specialized in interdisciplinary projects bringing together microsystems, materials technology, and optics. His activities are documented in over 200 publications with topics ranging from liquid crystal optics (book published by Wiley in 2006) to amorphous nanophotonics (book published in 2013). In 2020 he joined SUSS MicroOptics to participate in the fascinating technological developments in micro-optics. 